\title{
A New Mode Based on Battery Transportation to Reduce The Wind Curtailment
}

\author{
Han Jiang ${ }^{1, ~ a ~}$, Zhanlong Zhang ${ }^{1, b}$ and Guohua Liü \\ ${ }^{1}$ State Key Laboratory of Power Transmission Equipment \& System Security and New Technology, \\ Chongqing University, Chongqing, 400030, China \\ ${ }^{2}$ Quzhou Power Supply Company, State Grid Electric Power Company, Quzhou, Zhejiang Province, \\ 324000, China \\ ajianghan_wyq@outlook.com, 'bhangzl@cqu.edu.cn, ${ }^{9}$ lynn415@126.com
}

\begin{abstract}
Keywords: renewable energy, battery, transportation, penetration
Abstract. The development of renewable energy such as wind power has been widely concerned. However, the randomness and intermittency of the wind power as well as the lack of transmission capacity result in significant amount of wind energy curtailed. Meanwhile, the demand for large capacity battery is expanding from the user side owing to the rapid development in electric vehicles (EV) and plug-in hybrid EVs. Considering the issues mentioned above, a novel mode is proposed in which empty batteries are transported from battery exchange stations in high-load areas to wind farm. The proposed mode is described in detail. A corresponding mathematic model is established and a case study is presented. The result indicates that batteries for storing the otherwise curtailed energy can also be used to smooth the wind power output. This mode delivers a potential solution to the problem of curtailment and gives fresh impetus to the development of EV battery exchange mode.
\end{abstract}

\section{Introduction}

The energy and environment issues are becoming increasingly serious. As a result, more and more people around the world begin to focus on renewable energy which is clean and sustainable. However, areas with abundant renewable energy resources are often dispersed in remote locations where the local energy consumption is low and the transmission capacity is limited. In such regions, wind power and solar power curtailment, whereby power generation exceeds demand and production must be lowered, can be quite severe [1,2]. Take wind power in China as an example, wind resources are mainly concentrated in the north, while the load center is on the southeast coast. As the capacity of long-distance transmission lines cannot match the need, such distribution leads to a serious wind curtailment. In 2014 the wind power curtailment proportions in Jilin province, Heilongjiang province and Inner Mongolia were all over 20 percent and showed a rising trend [2]. Some means have been proposed to address the problem of wind curtailment such as using the pumped storage to improve the ability of wind power consumption [3] and increasing transmission line capacity [4]. These ways can alleviate the problem of wind curtailment to a certain extent, but site selection, long construction times and poor economic performance are issues. Besides, with the expansion of EV and PHEV industrial, the consumer demand of large capacity battery is increasing dramatically driving the development and competition in battery industrial. Battery performance such as specific capacity continues to improve, while the price of per kilowatt is rapidly decreasing. However, the follow-up problem is that the large-scale unplanned charging of EVs and plug-in hybrid EVs may cause a large and intermittent load. This unpredictable load could seriously affect the stability of the existing power grid [5]. Therefore, it is necessary to explore a more flexible solution with relatively short planning and construction period to solve the issues mentioned above. 


\section{Model Description}

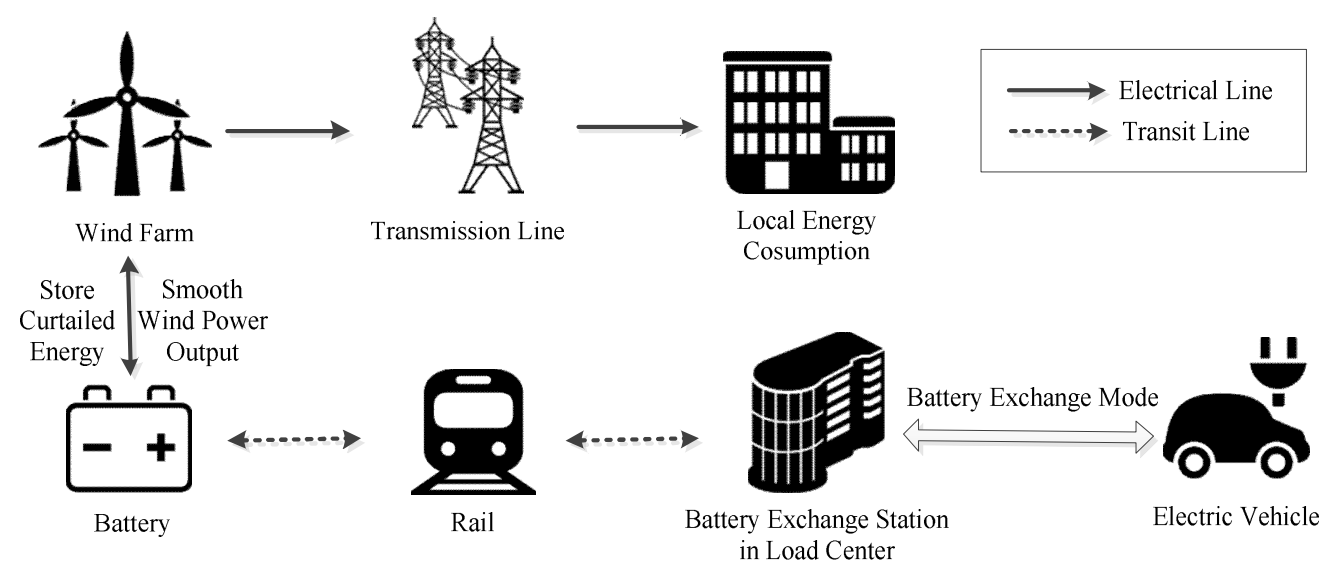

Fig. 1 Configuration of the mode

The present study uses a wind farm where wind curtailment occurs as an example (Fig. 1). By installing battery charging devices at the wind farm and using the railway or other low-cost transportation, the empty batteries from battery exchange stations in load center can be transported uniformly to wind farm and charged centrally. These batteries are utilized to store otherwise curtailed energy and smooth the unstable wind power output at the same time. Different from the typical battery energy storage system of which the batteries are fixed, the battery models in this mode can be detached from the charging facilities and delivered back to the load canter once fully charged.

If realized, capture and use of otherwise curtailed energy would increase the cost effectiveness of wind farms, enabling wind power to compete better with other forms of power generation in the electricity market. For the consumer, this mode could encourage the deployment of battery exchanges, reducing waiting times for charging, and thereby improving efficiency. If rail is adopted for battery transportation, the whole generation-transportation-usage process could run on $100 \%$ renewable energy, which is beneficial for the environment. This mode could be applied to any renewable power plant that experiences oversupply and subsequent curtailment.

\section{Battery Charging Container}

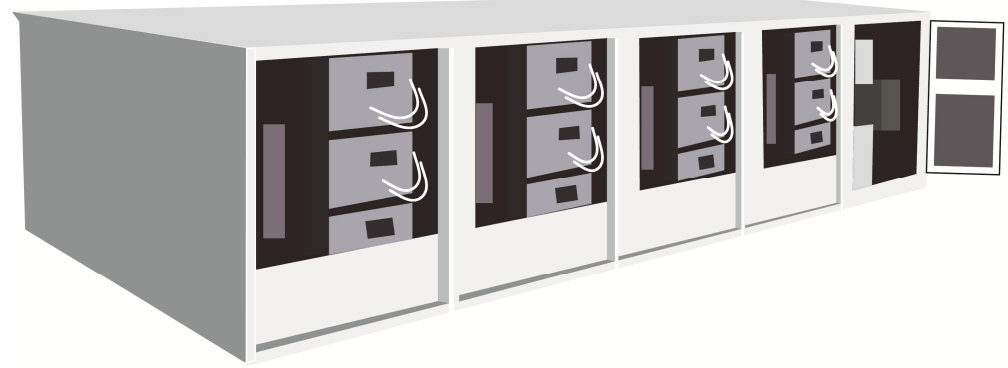

Fig. 2 Example of a battery charging container

In fact, the batteries available in the market have a wide range of specifications. Without a uniform standard, it would be almost impossible to manage the charging process with a wide variety of small-capacity batteries at the wind farm. According to the hypothesis of this paper, the proposed solution is that each battery manufacturing company should design their own battery charging containers (Fig. 2). The internal structure of these battery charging containers can be different according to divergent battery types. But the external characteristics in terms of the size, voltage, energy capacity and power must be in same. Therefore, these standardized battery charging containers can be uniformly managed for charging and transported between the wind farm and load center. 


\section{Control principle}

Wind curtailment occurs when some wind turbine generations (WTG) in a wind farm are required to reduce power output or to cease operating, even under normal operating conditions. The reasons for curtailment are complicated and specified curtailment commands are given through automatic generation control in real time. To simplify, the wind curtailment index is assumed as $K_{c}$ in this paper

$$
K_{c}=\frac{P_{a}}{P_{r}},
$$

where $P_{a}$ is the maximum acceptable power during the period, and $P_{r}$ is the rated power output of the wind farm. When the actual power output $P_{w}$ is larger than $P_{a}$, the batteries are responsible for covering the difference between them instantaneously. This part of the BESS power output is denoted as $P_{B_{-} \text {curt }}$.

$$
\begin{aligned}
P_{B_{-} \text {curt }} & =\left\{\begin{array}{cc}
P_{a}-P_{w} & P_{w}>P_{a} \\
0 & P_{w} \leq P_{a}
\end{array},\right. \\
P_{s} & = \begin{cases}P_{a} & P_{w}>P_{a} \\
P_{w} & P_{w} \leq P_{a}\end{cases}
\end{aligned}
$$

In (3), $P_{\mathrm{s}}$ represents the power output after the subtraction of the curtailed energy. Owing to wind instability, $P_{\mathrm{s}}$ fluctuates and may negatively affect stable operation of the grid. Therefore, it is essential to use the batteries for smoothing at the same time. In this paper, a first-order low-pass filter is adopted to smooth the power output, making the final power output $P_{f}$ conform to the power quality standard of the grid [6]. Fig. 3 shows the block diagram of the battery power output. Assuming the time constant of the low-pass filter is $T$, then $P_{f}$ and the smoothing part of battery power output is given as

$$
\begin{gathered}
P_{f}(s)=\frac{1}{1+s T} P_{s}(s), \\
P_{B_{-} s m o o t h}(s)=P_{f}(s)-P_{s}(s)=\frac{-s T}{1+s T} P_{s}(s) .
\end{gathered}
$$

By adding $P_{B_{-} \text {curt }}$ and $P_{B_{-} \text {smooth }}$, the final power output reference $P_{\text {ref }}$ can be obtained as

$$
P_{B_{-} \text {curt }}(t)+P_{B_{-} \text {smooth }}(t)=P_{\text {ref }}(t)
$$

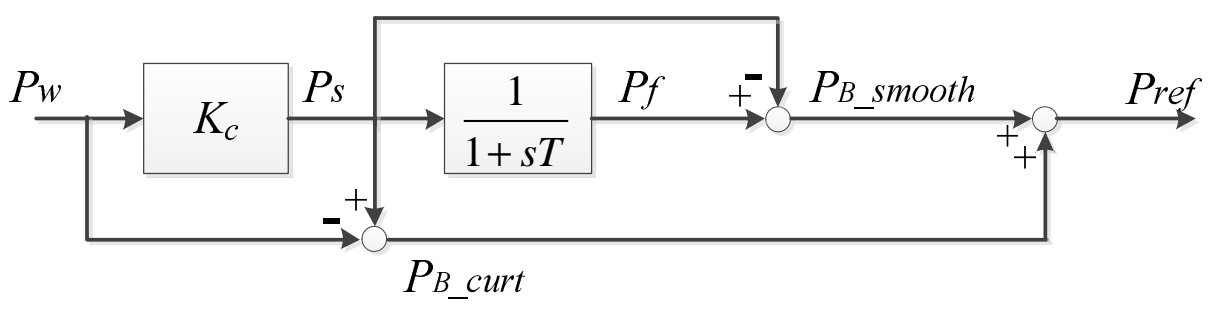

Fig. 3 Block diagram of the battery power output

\section{Case Study}

In this paper, the mathematic model of WTG is established and the parameters is shown in Table 1. To simplify, a 24-h scheduling period is taken as an example, with a constant wind curtailment level. The curtailment index $K_{c}$ is assumed to be 0.6 . The 24-h wind speed data from a wind farm is adopted. 
Table 1. Parameters of the wind turbine generator

\begin{tabular}{cc}
\hline Wind turbine parameter & Value \\
\hline Rated power & $2.5[\mathrm{MW}]$ \\
Cut-in speed & $4[\mathrm{~m} / \mathrm{s}]$ \\
Cut-out speed & $25[\mathrm{~m} / \mathrm{s}]$ \\
Rated speed & $12[\mathrm{~m} / \mathrm{s}]$ \\
Blade radius & $52.77[\mathrm{~m}]$ \\
$C_{P}$ & 0.27 \\
\hline
\end{tabular}

The results are shown in Table 2 . In this case, the battery capacity used to store the curtailed energy is sufficient for power smoothing. Overall, the required battery energy capacity is $2.9359 \mathrm{MWh}$ and the required power is $1.1626 \mathrm{MW}$.

Table 2. Simulation results

\begin{tabular}{cc}
\hline Parameter & Value \\
\hline Total output energy & $22.3687[\mathrm{MWh}]$ \\
Curtailed energy & $2.9359[\mathrm{MWh}]$ \\
Curtailment ratio & $13.12[\%]$ \\
Capacity in need for smoothing & $0.0712[\mathrm{MWh}]$ \\
$P_{B_{-} \text {smooth }}(\max )$ & $0.7653[\mathrm{MW}]$ \\
$P_{B_{-} \text {curt }}(\max )$ & $1[\mathrm{MW}]$ \\
$P_{\text {ref }}(\max )$ & $1.1626[\mathrm{MW}]$ \\
\hline
\end{tabular}

\section{Conclusion}

The present study proposed that wind farms use large quantities of discharged batteries to store the energy that would otherwise be curtailed. Batteries would be transported between wind farms and load centers via rail or other low-cost means. The proposed mode is described in detail and the concept of battery charging container is also presented. The result of a case study based on the corresponding mathematic model indicates that the batteries used to store the curtailed energy can also smooth the wind power output. The economic analysis such as the cost of transportation will be considered in the upcoming research. This new mode might improve the penetration of wind power and provide insights into how to promote renewable energy in the future.

\section{References}

[1] S. Martín-Martínez, E. Gómez-Lazaro, A. Molina-Garcia and A. Honrubia-Escribano, 2014 IEEE PES General Meeting | Conference \& Exposition, National Harbor, MD, 1-5. (2014)

[2] Wang Zhaobin, Energy (In Chinese) 7, 42. (2014)

[3] A. B. T. Attya and T. Hartkopf, IET Generation, Transmission \& Distribution 9(12), 1485. (2015)

[4] C. Kang et al, IEEE Power and Energy Magazine 11(5), 56. (2013)

[5] L. Jian, H. Xue, G. Xu, X. Zhu, D. Zhao and Z. Y. Shao, IEEE Transactions on Industrial Electronics 60(8), 3218. (2013)

[6] China State Grid Electric Power Company Enterprise Standards: Q/GDW392-2009, Regulations on accessing power system technology to wind electric field in grid. (2009) 